\title{
Cross-Database Evaluation Using an Open Finger Vein Sensor
}

\author{
Matthias Vanoni, Pedro Tome, Laurent El Shafey and Sébastien Marcel \\ Idiap Research Institute \\ Centre du Parc, Rue Marconi, 19, CH-1920, Martigny, Switzerland \\ \{matthias.vanoni, pedro.tome, laurent.el-shafey, sebastien.marcel\}@idiap.ch
}

\begin{abstract}
Finger vein recognition is a recent biometric application, which relies on the use of human finger vein patterns beneath the skin's surface. While several methods have been proposed in the literature, its applicability to uncontrolled scenarios has not yet been shown. To this purpose this paper first introduces the VERA database, a new challenging publicly available database of finger vein images. This corpus consists of 440 index finger images from 110 subjects collected with an open device in an uncontrolled way. Second, an evaluation of state-ofthe-art finger vein recognition systems is performed, both on the controlled UTFVP database and on the new VERA database. This is achieved using a new open source and extensible finger vein recognition framework, which allows fair and reproducible benchmarks. Experimental results show that challenging recording conditions such as misalignments of the fingers lead to an absolute degradation in equal error rate of $2.75 \%$ up to $24.10 \%$ on VERA when compared to the best performances on UTFVP.
\end{abstract}

\section{INTRODUCTION}

Finger vein recognition is a fairly new topic in the biometric landscape, which utilizes the vein patterns inside a person's finger [1]. Compared to other biometric modalities such as fingerprint, it has the advantage of being contactless. Besides, it is considered as being difficult to forge, since vein patterns are hidden beneath the skin [1].

The idea of employing the vascular patterns embodied in human fingers for authentication purposes originally comes from Hitachi Research Laboratory at the end of the nineties [2]. While studying human brain's blood system using imaging techniques, they discovered that near-infrared (NIR) light is absorbed significantly more by hemoglobin than by the surrounding tissues. Furthermore, transmitting NIR light around $850 \mathrm{~nm}$ through the finger appears to be a suitable technique to image the vein patterns [3]. The first patent application to finger vein recognition was, hence, submitted by Hitachi Ltd. in 2001 and obtained in 2004 [4]. Commercial physical access control devices based on finger vein were made available in 2002 and logical access for ATMs in 2005 [2]. Nowadays this technology is widely used in the financial sector in Japan, China and Poland, where it proved to be accurate. Very recently the technology has been introduced in hospitals in Turkey for patient identification. Other potential usage in the medical domain can be access control for electronic medical records (EMR) systems, access control for medical and chemical storage facilities, patient verification to prevent wrong-patient surgery or patient verification in remote medical services.
Although finger vein authentication is already commercially deployed, its scientific foundation is still quite limited due to industrial protectiveness [5]. As a consequence, research on finger vein recognition requires both to develop and build sensors as well as to collect images (see Table I) before being able to investigate the use of suitable preprocessing and pattern recognition techniques. Therefore, this has been a limiting factor for researchers to propose novel and robust finger vein recognition approaches, as well as to fairly benchmark stateof-the-art approaches. Interestingly, the University of Twente released an open device and an open database (UTFVP) enabling for research on finger vein recognition [6].

The novelty and the main contributions of this paper can be summarized by:

- A reproducible cross-database evaluation of state-ofthe-art techniques.

- VERA database: a new challenging publicly available database acquired with the same open sensor as the UTFVP database.

- A new open source and extensible finger vein recognition framework publicly available for the research community.

The remainder of this paper is structured as follows: Section 2 presents an overview of existing finger vein databases and describes the collected database. Section 3 reports our experiments and results based on open source implementations of state-of-the-art algorithms. Finally, Section 4 concludes the paper.

\section{Finger VEIN DATABASES}

Due to the technological challenges in acquiring finger vein images of sufficient quality the amount of available databases is scarce.

\section{A. Overview of Existing Databases}

Table I provides an overview of the finger vein databases used in the last ten years by the industry and the research community. In particular, it can be noticed that such databases are publicly available only since fairly recently.

To the best of our knowledge, Hitachi Ltd and Mofiria Ltd are the only two companies providing fingervein solutions for biometric applications. Their patented technologies and nondisclosed databases has lead researchers to create their own 


\begin{tabular}{|c|c|c|c|c|c|c|c|c|c|c|}
\hline Databases & Available & \#Subjects & $\begin{array}{c}\text { Fingers } \\
\text { per subject }\end{array}$ & \#Sess. & $\begin{array}{l}\text { \#Acq. per } \\
\text { session }\end{array}$ & \#Images & $\begin{array}{c}\text { Image } \\
\text { size }\end{array}$ & Device & Year & $\operatorname{EER}(\%)$ \\
\hline Hitachi Res. Lab. & NO & 2,673 & $\overline{L_{i, m}, R_{i, m}}$ & 1 & 11 & 117,612 & $?$ & TS-E3F1 & 2004 & 0.0 [7] \\
\hline Int. Biom. Group & NO & 650 & $L_{i, m}, R_{i, m}$ & 2 & $2-9$ & 28,600 & $?$ & TS-E3F1 & 2006 & $0.5[7]$ \\
\hline Hitachi-Kyushu & NO & 506 & $R_{i}$ & 1 & 2 & 1,012 & $?$ & TS-E3F1 & 2007 & $0.0[8]$ \\
\hline PKU v.2,3,4 & YES & 5,208 & $L_{i, l}, R_{i, l}$ & 1 & 5 & 50,700 & $512 \times 384$ & Proto PKU & 2008 & $1.2[9]$ \\
\hline GUC45 & NO & 45 & 10 & 12 & 2 & 10,800 & $512 \times 240$ & Proto GUC & 2009 & $0.7[10]$ \\
\hline SDUMLA-HMT & YES & 106 & $L_{i, m, r}, R_{i, m, r}$ & 1 & 6 & 3,816 & $320 \times 240$ & Proto Wuhan Univ. & 2010 & - \\
\hline HKPU & YES & 156 & $L_{i, m}, R_{i, m}$ & 2 & 6 & 6,264 & $513 \times 256$ & Proto HKPU & 2011 & $0.6[11]$ \\
\hline UTFVP & YES & 60 & $L_{i, m, r}, R_{i, m, r}$ & 1 & 4 & 1,440 & $672 \times 380$ & Proto Twente Univ. & 2013 & $0.4[6]$ \\
\hline MMC BNU6000 & YES & 100 & $L_{i, m, r}, R_{i, m, r}$ & 1 & 10 & 6,000 & $640 \times 480$ & Proto Chonbuk Univ. & 2013 & - \\
\hline CFVD & YES & 13 & $L_{i, m, r}, R_{i, m, r}$ & 2 & 51 & 1,345 & $640 \times 480$ & Proto Shandong Univ. & 2013 & - \\
\hline Shandong Univ. & NO & 34 & $L_{i, m}, R_{i, m}$ & 2 & $20-10$ & 4,080 & $320 \times 240$ & Proto Whuan Univ. & 2013 & $1.1[12]$ \\
\hline FV-USM & YES & 123 & $L_{i, m, r}, R_{i, m, r}$ & 2 & 6 & 5,904 & $640 \times 480$ & Proto Sains Univ. & 2013 & $2.3[13]$ \\
\hline VERA & YES & 110 & $\mathbf{L}_{\mathbf{i}}, \mathbf{R}_{\mathbf{i}}$ & 1 & 2 & 440 & $665 \times 250$ & Proto Twente Univ. & 2014 & 6.2 \\
\hline
\end{tabular}

TABLE I. OVERVIEW OF FINGER VEIN DATABASES. THIS TABLE SUMMARIZES THE PROPERTIES OF SEVERAL FINGER VEIN DATABASES. THE FINGERS PER SUBJECT COLUMN SPECIFIES WHICH FINGERS WERE USED AMONG $(R)$ IGHT OR $(L)$ EFT HAND, $(t)$ HUMB, $(i)$ NDEX, $(m)$ IDDLE, $(r)$ ING, AND $(l)$ ITTLE FINGER. PROTO STANDS FOR LABORATORY-MADE PROTOTYPE. ALL DATABASES ARE ACQUIRED BY TRANSMISSION EXCEPT CFVD THAT WAS ACQUIRED BY REFLECTION.

prototypes and databases as a preliminary step before testing the technology and measuring its performances. This work started in 2008 with the Peking University (PKU) achieving an EER of $1.2 \%$ compared to the almost $0 \%$ from the industry. Between 2008 and 2011 the PKU collected around 60,000 images of finger veins from index and middle fingers and made 11,650 of them publicly available [9]. Meanwhile, in 2010 the University of Shandong released the first finger vein database embracing six different fingers from 106 users [14]. They also published a one of a kind finger vein database obtained by reflecting the NIR light on the fingers rather than transmitting it through the fingers [15]. In 2013, the University of Twente released a controlled database of high quality (UTFVP) using an open finger vein sensor [5]. Besides, they compare the performance of several state-of-the-art algorithms both on the UTFVP and on the PKU databases [6].

To the best of our knowledge, reproducible results on finger vein recognition were only published in [6], from the description of an open finger vein device and an open database, to the release of an open source MATLAB-based framework. ${ }^{1}$ This open finger vein sensor described in [5] and the UTFVP database were chosen as a starting point for our work. Furthermore, this paper introduces a new database based on this open finger vein sensor previously tested in the literature.

\section{B. VERA: A Challenging Finger Vein Database}

The new VERA database consists of 440 finger vein images from 110 subjects recorded in an uncontrolled way in terms of finger alignment. In addition, subjects are from various ethnicities with a ratio female:male of 40:70, which brings an additional challenge, since skin properties affect the acquisition of vascular patterns (see Figure 1).

A recent study [11] showed that the index is the most discriminative finger when compared to the other four fingers. However, a different conclusion was reached on the UTFVP database in [5], the authors observing that finger vein recognition using index fingers is less accurate than when using ring or middle fingers. Although further investigations are required to understand these differences, we decided to collect only index fingers for this first phase of the VERA database.

\footnotetext{
${ }^{1}$ http://www.mathworks.ch/matlabcentral/fileexchange/index?term= authorid $\backslash \% 3 \mathrm{~A} 57311$
}
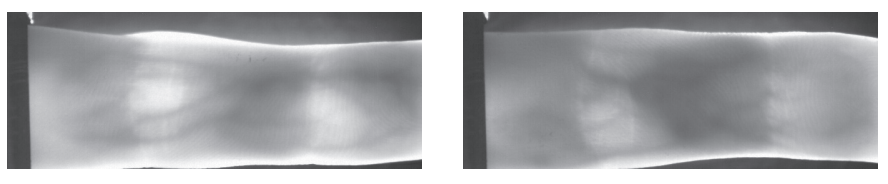

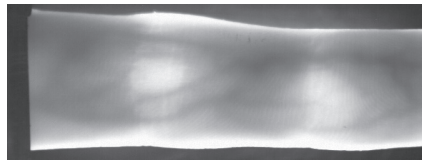

(a) Left index fingers (male)
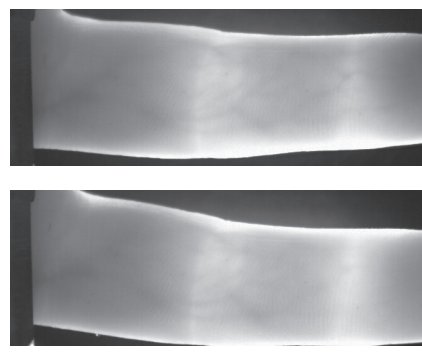

(c) Left index fingers (female)

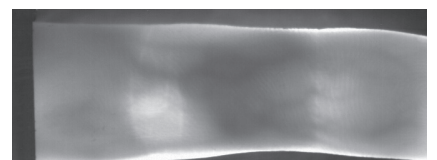

(b) Right index fingers (male)

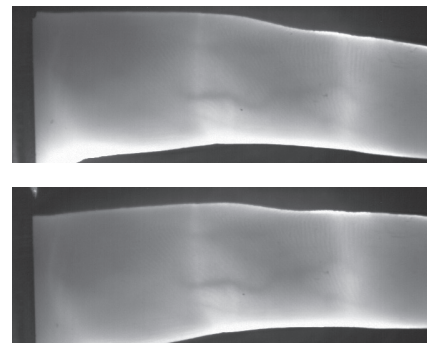

(d) Right index fingers (female)
Fig. 1. SAmples from the VerA Database. This figure depicts finger vein images of left $((a)$ and $(c))$ and right $((b)$ and $(d))$ index fingers from a male $((a)$ and $(b))$ and a female $((c)$ and $(d))$ subjects.

Data acquisition, hence, turns into a quick and friendly user experience, which emulates a realistic scenario. In practice, two shots of each index finger are collected from each subject in a single session. The acquisition sensor used for the collection is the open sensor described in [5]. Figure 1 depicts all available images from two specific subjects (one female and one male) of the VERA database. Finger vein images and metadata that include age and gender information are publicly and freely available. ${ }^{2}$

Although the images from the VERA database are obtained with the same device as the images from the UTFVP database, their overall appearance, shape and quality differ (see Figure $2(a)$ and $(d)$ ). These differences mainly come from a slight misalignment of the internal mirror and a different crop of the region of interest in the acquisition sensor. As shown on Figure 2, this directly impacts the preprocessing step of the finger vein recognition system. As a consequence, finger

\footnotetext{
${ }^{2}$ http://www.idiap.ch/dataset
} 

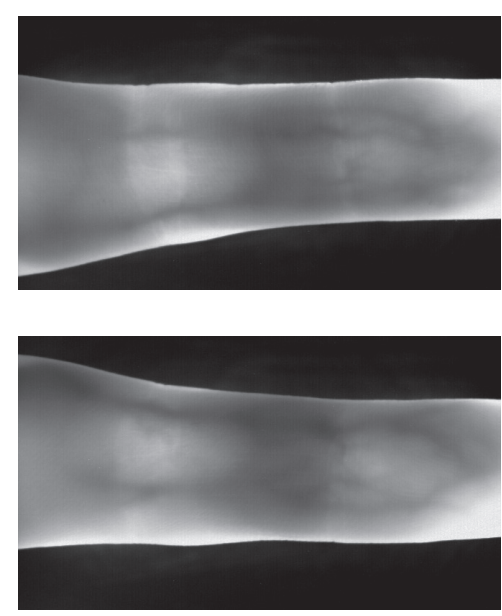

(a) UTFVP DB original (controlled)
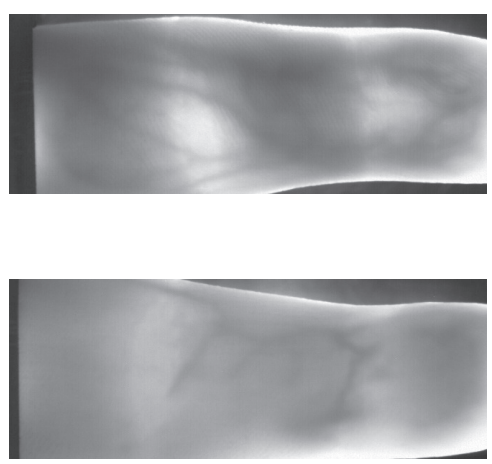

(d) VERA DB original (uncontrolled)
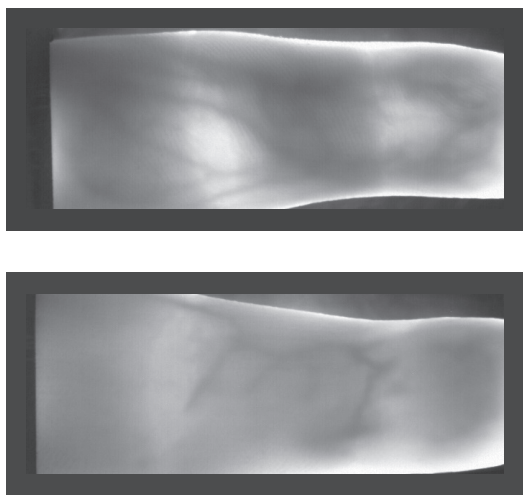

(g) VERA DB original + Paddy array (uncontrolled)

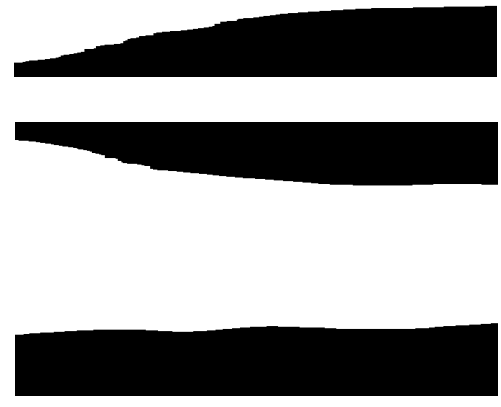

(b) UTFVP DB

Finger cropped mask
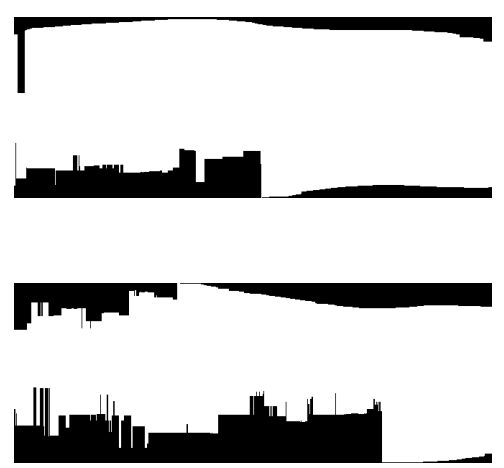

(e) VERA DB

Finger cropped mask
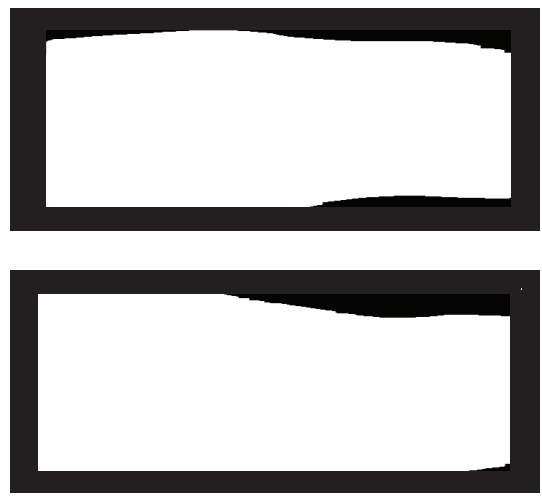

(h) VERA DB

New finger cropped mask
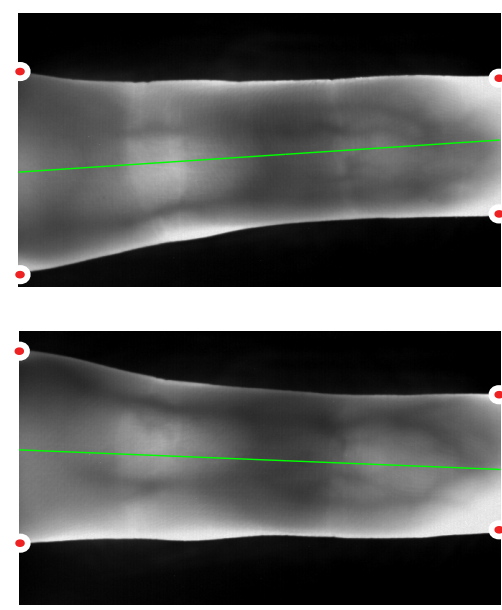

(c) UTFVP DB

Pattern normalization
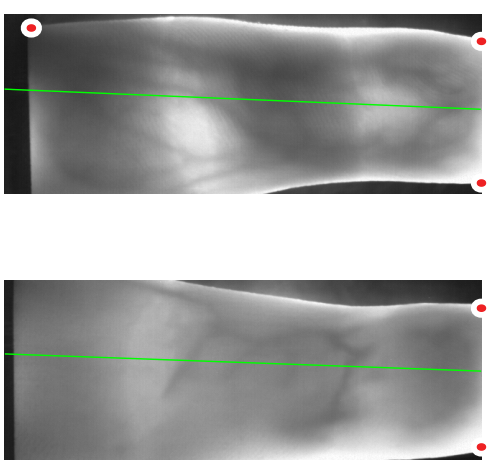

(f) VERA DB

Pattern normalization
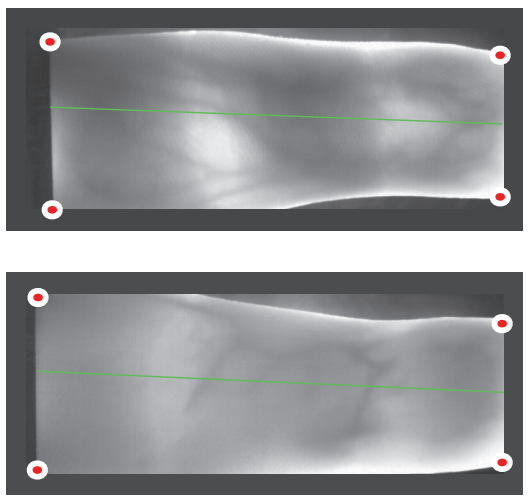

(i) VERA DB

Pattern normalization + Paddy array

Fig. 2. COMPARISON OF THE PREPROCESSING ON THE UTFVP AND VERA DATABASES. This figure shows sample images from the UTFVP $(672 \times 380$, $(a))$ and the VERA $(665 \times 250,(d)$ and $(g))$ databases, as well as the finger cropped masks $((b),(e)$ and $(h))$ and the normalized patterns $((c),(f)$ and $(i))$ obtained after preprocessing these images. Last rows show the preprocessing results of the new segmentation applied to VERA database. 
vein images from the VERA database are more challenging in terms of segmentation, alignment and recognition.

Therefore, the VERA database is useful for measuring the generalization of algorithms. In particular, robust preprocessing and feature extraction methods could be tested both on the UTFVP and VERA databases to better tackle cross-database issues. In the next section, we follow this line of research considering a set of three state-of-the-art feature extraction techniques.

\section{EXPERIMENTS AND RESULTS}

This section describes the baseline systems, the methodology followed, the evaluation protocols, and the experimental results achieved in this work.

\section{A. Baseline Systems}

The experiments in this paper are carried out using the open source finger vein framework called FingerveinRecLib: xbob.fingervein. ${ }^{3}$ This framework is extensible and allows to run a complete finger vein recognition experiment, from the preprocessing of raw images to the computation of biometric scores and their evaluation.

The preprocessing step consists of two different configurations (with or without histogram equalization - Heq), Heq being first applied if required. Next, the contours of the fingers are localized using edge detection filter masks as described in [16]. The finger image is then normalized by fitting a straight line between the detected finger edges, whose parameters (a rotation and a translation) are used to create an affine transformation [9]. Figure 2 illustrates this process. Detecting properly the finger edges is more challenging on the VERA database and applying the proposed segmentation from Ton et al on UTFVP leads to poor results. To handle that issue a new segmentation method has been implemented on VERA and made available in our open source framework. Figure 2 shows the improvement in terms of finger segmentation.

Next, feature extraction is performed, which aims at emphasizing the vein patterns in the image. Three different stateof-the-art approaches for finger vein recognition are currently implemented in this framework: $i$ ) maximum curvature [17], ii) repeated line tracking [18], and iii) wide line detector [9].

Once feature extraction is completed, the resulting finger vein images are compared using a simple noncommutative template matching algorithm initially proposed in [18]. This technique computes the maximum correlation between the two input templates while allowing limited vertical and horizontal displacements. The performance of the resulting systems is measured in terms of Equal Error Rate (EER) and Detection Error Tradeoff (DET) curves [19].

Since there are two different preprocessing configurations and three feature extraction techniques, this finally leads to six different systems that are evaluated in the remainder of this section.

\footnotetext{
${ }^{3}$ Freely available at http://pypi.python.org/pypi/xbob.fingervein
}

\begin{tabular}{|c|c|c|c|c|}
\hline Database & Protocol & \#Fingers & $\begin{array}{c}\text { \#Genuine } \\
\text { scores }\end{array}$ & $\begin{array}{c}\text { \#Impostor } \\
\text { scores }\end{array}$ \\
\hline \hline \multirow{3}{*}{ UTFVP DB } & Full $l_{\text {train }}$ & 35 & 210 & 6,720 \\
& $F u l l_{\text {test }}$ & 325 & 3,900 & $1,684,800$ \\
\cline { 2 - 5 } & $B_{\text {train }}$ & 35 & 210 & 6,720 \\
& $B_{\text {test }}$ & $\mathbf{1 0 8}$ & $\mathbf{2 1 6}$ & $\mathbf{4 6 , 2 2 4}$ \\
\hline \hline & $F u l l_{\text {train }}$ & 0 & 0 & 0 \\
VERA DB & $F u l l_{\text {test }}$ & 220 & 440 & 192,720 \\
\cline { 2 - 5 } & $B_{\text {train }}$ & 112 & 224 & 49,728 \\
& $B_{\text {test }}$ & $\mathbf{1 0 8}$ & $\mathbf{2 1 6}$ & $\mathbf{4 6 , 2 2 4}$ \\
\hline
\end{tabular}

TABLE II. EVALUATION PROTOCOLS. THIS TABLE REPORTS THE PROTOCOL.

\section{B. Methodology}

The methodology employed in this study is based on the following three steps:

1) We reproduce the experiments on the so-called Full evaluation protocol of the UTFVP database and compared our performance with the one obtained in [6].

2) We evaluate the systems on the VERA database using the so-called Full evaluation protocol, which is defined in the same way as on UTFVP.

3) We evaluate the systems separately on the UTFVP and the VERA databases using an evaluation protocol called $B$ that is designed to involve the same fingers, the same number of fingers and the same number of scores.

The definition of these evaluation protocols is provided in the next subsection.

\section{Evaluation Protocols}

The different evaluation protocols used in this paper are summarized in Table II. For each protocol, the database is divided into two sets: training (for tuning the parameters of the systems) and test (for reporting the accuracy of the systems). In this study, we adopted the optimal parameter values tuned in [5] on the UTFVP database, and the training sets are, hence, not used in our experiments. Matching scores are computed using the samples of the test set in an exhaustive way, considering all possible ordered pairs of samples.

The Full evaluation protocol on UTFVP corresponds to the one originally defined in [5]. The corresponding test set consists of 325 fingers $\left(L_{i, m, r}\right.$ and $\left.R_{i, m, r}\right)$ from 60 subjects, while the remaining 35 fingers are parts of the training set.

On the VERA database, we define a similar Full evaluation protocol by considering all the 220 collected fingers $\left(L_{i}\right.$ and $R_{i}$ ) from the 110 subjects as a test set, which results in 440 genuine and 192, 720 impostor scores.

To allow a fair comparison of the performance on both databases, a new protocol called $B$ has been defined. It involves the same fingers, the same number of fingers and the same number of scores on both databases. On the UTFVP database, the test set consists of keeping only the first two (out of four) samples of the index fingers (hence, discarding all the samples from non-index fingers), while the training set remains unchanged. On the VERA database, the test set is comprised of the following samples for similarity purposes: the images of the left and right index fingers of the first 48 subjects, 


\begin{tabular}{|c|c|c|c||c|c|}
\hline \multicolumn{2}{|c|}{ Approach } & \multicolumn{2}{c|}{ UTFVP DB } & \multicolumn{2}{c|}{ VERA DB } \\
\cline { 3 - 6 } & Full $[6]$ & $B$ & $B$ & Full \\
\hline \hline \multirow{2}{*}{ Maximum curvature } & Heq & $\mathbf{0 . 4 6}$ & 1.85 & $\mathbf{3 . 2 4}$ & $\mathbf{6 . 2 0}$ \\
& - & 0.49 & $\mathbf{1 . 3 9}$ & 4.17 & 5.53 \\
\hline \multirow{2}{*}{ Repeated line tracking } & Heq & 1.05 & 1.85 & 27.78 & 28.10 \\
& - & $\mathbf{0 . 7 4}$ & $\mathbf{1 . 8 2}$ & $\mathbf{2 5 . 9 3}$ & $\mathbf{2 6 . 7 8}$ \\
\hline \multirow{2}{*}{ Wide line detector } & Heq & $\mathbf{0 . 7 4}$ & $\mathbf{0 . 9 2}$ & 12.04 & 11.28 \\
& - & 1.56 & 1.85 & $\mathbf{1 1 . 1 1}$ & $\mathbf{1 1 . 7 3}$ \\
\hline
\end{tabular}

TABLE III. SYSTEM PERFORMANCE (EER IN \%). THIS TABLE REPORTS THE SYSTEM PERFORMANCE (EER IN \%) FOR PROTOCOLS Full AND $B$ ON BOTH DATABASES. Heq INDICATES WHETHER HISTOGRAM EQUALIZATION IS PERFORMED DURING THE PREPROCESSING STEP OR NOT. THE BEST RESULTS OF EACH METHOD ARE HIGHLIGHTED IN BOLD.

the left index finger of the next 6 subjects (subject id 49 to 54 ) and the right index fingers of the next 6 subjects (subject id 55 to 60 ). Remaining images are assigned to the training set. This $B_{\text {train }}$ set is not used in this paper as we consider only the parameters optimised on the UTFVP training set and evaluate cross-databases performance of three different fingervein recognition algorithms.

\section{Experimental Results}

The performance (EER in \%) of the six considered finger vein recognition systems on the two databases is summarized in Table III. In the following, these experimental results are discussed according to the structure of the methodology section.

1) System performance on the UTFVP database: Considering the UTFVP Full protocol, the results reported in [6] have been successfully reproduced as shown in Table III (first column). This means that our implementation of the baseline systems is legitimate to report performance on other databases such as the new VERA database.

2) System performance on the VERA database: The system performance on the Full protocol of the VERA database is shown in the last column of Table III. Overall, the reported EER are significantly larger than the ones previously obtained on the UTFVP database.

The best results are obtained using the feature extraction technique based on maximum curvature while applying histogram equalization during the preprocessing stage $(6.20 \%$ of EER). Besides, it is interesting to note that the two other feature extraction techniques, repeated line tracking and wide line detector approaches, respectively, lead to very different results. The wide line detector approach achieved an EER of $11.28 \%$ when histogram equalization is performed, while the repeated line tracking approach provides the worst results (28.10\% of EER).

These results suggest that VERA is a challenging database in terms of preprocessing, alignment and recognition.

3) Comparison between both databases: A fair comparison between both databases can be carried out using the previously defined $B$ evaluation protocol, which only considers the index fingers and results in the same number of genuine and impostor scores on both databases. The results in terms of EER are summarized in Table III. Additionally, DET curves of the systems (considering the best preprocessing configuration) are reported in Figure 3.
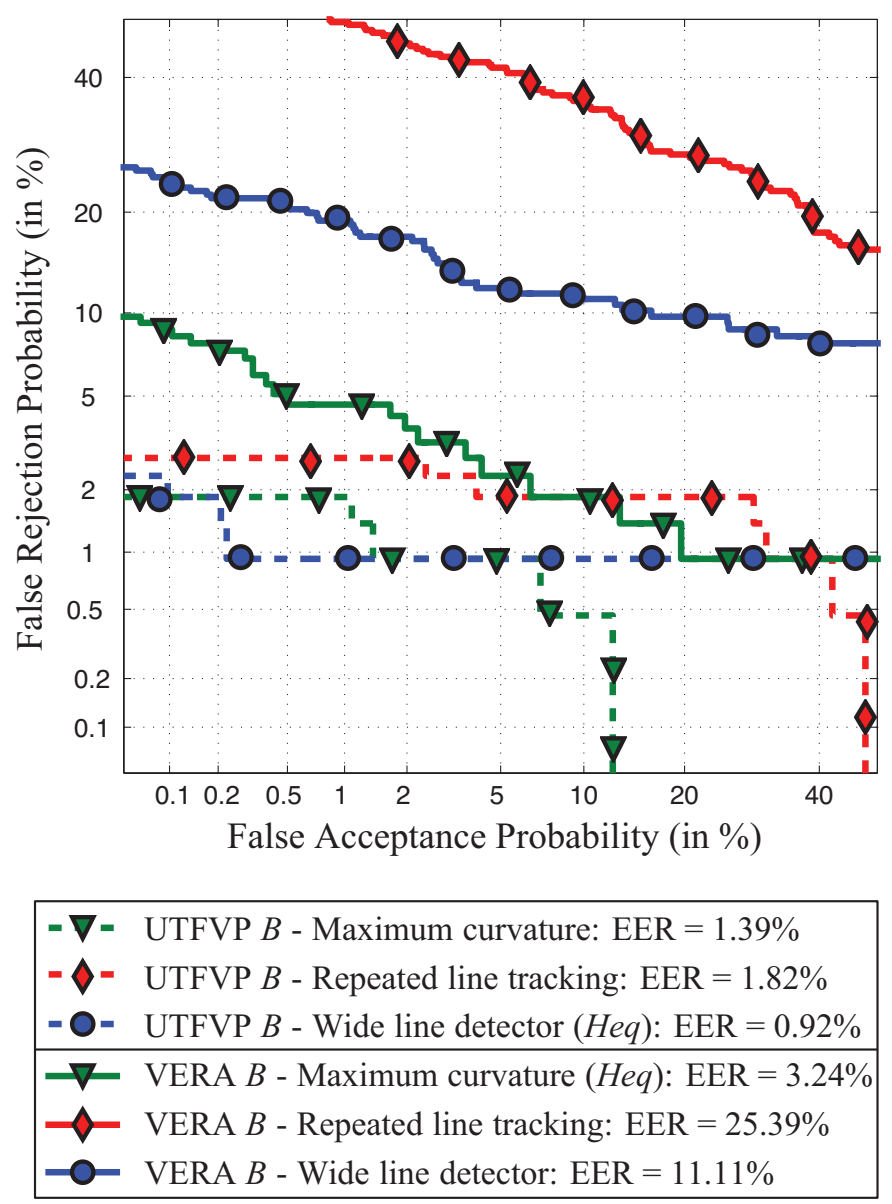

Fig. 3. DET CURVES OF THE SYSTEMS. This figure shows the DET curves of the systems on the UTFVP and VERA databases considering the best preprocessing configuration. For more details check the Table III.

Comparing the results on both databases, we clearly notice the difference between the two databases and the lack of generalisation from UTFVP to VERA. Figure 3 shows that the performance of the maximum curvature approach with histogram equalization is less impacted by the difficult conditions encountered in the VERA database than the one of the other systems. Besides, performances reported previously in the literature (see Table I) are significantly better than the ones obtained on this new and challenging VERA database (e.g. $0.5 \%$ on Int. Biom. Group vs. $3.24 \%$ on VERA). This gap may be explained by the quality and misalignment of the recorded images.

Considering the UTFVP database, it is interesting to note that the systems achieve a high verification rate, even when they are operating at a high security level (FAR $\left.<10^{-4}\right)$. In contrast, results on the VERA database suggest that current finger vein recognition approaches perform poorly, when the variability of the samples is large.

Despite the high variability of the samples from the VERA database, the notion of failure to enroll is not taken into account in our work, since no quality assessment has been implemented. This may lead to the acquisition of images with hardly visible veins, affecting the overall performance of the systems. For instance, we noticed during the acquisition phase 
that both women and people with dark skin are more likely to deliver finger vein images of poor quality. In addition, we recall that this cross-database evaluation relies on parameter values for feature extraction and matching that were optimized on the UTFVP database in [5]. Therefore, this may results in suboptimal performance when the systems are evaluated on the VERA database.

Overall, the conducted evaluation shows that finger vein recognition technology is still in a growing stage: While existing systems are very accurate in laboratory-like conditions, there is still room for improvement in realistic scenarios where the user tries to authenticate as quickly as possible with little cooperation effort. To this end, the VERA database is an interesting resource for the research community.

\section{CONCLUSIONS}

This paper presents a reproducible cross-database evaluation using an open finger vein sensor. In particular, a new publicly available finger vein database called VERA is introduced. Images were collected using the same open device as for the UTFVP database. However, the VERA database is voluntarily challenging in terms of misalignment and variability of the samples to emulate realistic scenarios.

In addition, this works comes with an open source and extensible finger vein recognition framework to foster reproducible and further research. Using this framework, experiments are conducted both on the UTFVP and the less controlled VERA databases. Experimentally, finger vein recognition appear to be very accurate in a controlled environment. However, generalization of state-of-the-art algorithms remains an issue, our cross-database evaluation resulting in an absolute degradation in equal error rate of $2.75 \%$ up to $24.10 \%$ on the challenging VERA database when compared to the best performances on UTFVP.

\section{ACKNOWLEDGMENT}

The authors would like to thank Prof. R. Veldhuis for sharing its finger vein sensor, as well as the EU FP7 BEAT (284989) project and the Swiss Centre for Biometrics Research and Testing for support.

\section{REFERENCES}

[1] A. K. Jain, P. Flynn, and A. A. Ross, Handbook of Biometrics, 2007.

[2] "Finger vein authentication: White paper," Hitachi, Ltd., Tech. Rep., 2006.

[3] M. Kono, H. Ueki, and S.-I. Umemura, "Near-infrared finger vein patterns for personal identification," Applied Optics, vol. 41, no. 35, pp. 7429-7436, Dec 2002.

[4] M. Kono, S. Umemura, T. Miyatake, K. Harada, Y. Ito, and H. Ueki, "Personal identification system," 2004, uS Patent 6,813,010. [Online]. Available: https://www.google.com/patents/US6813010

[5] B. Ton, "Vascular pattern of the finger: biometric of the future? sensor design, data collection and performance verification," Master's thesis, University of Twente, July 2012.

[6] B. Ton and R. Veldhuis, "A high quality finger vascular pattern dataset collected using a custom designed capturing device," in IEEE International Conference on Biometrics (ICB), 2013, pp. 1-5.

[7] M. Himaga and K. Kou, "Finger vein authentication technology and financial applications," in Advances in Biometrics. Springer, 2008, pp. $89-105$.
[8] T. Yanagawa, S. Aoki, and T. Ohyama, "Human finger vein images are diverse and its patterns are useful for personal identification," Kyushu University, MHF Preprint Series 12, 2007.

[9] B. Huang, Y. Dai, R. Li, D. Tang, and W. Li, "Finger-vein authentication based on wide line detector and pattern normalization," in International Conference on Pattern Recognition (ICPR), 2010, pp. 1269-1272.

[10] D. Hartung, "Vascular pattern recognition: And its application in privacy-preserving biometric online-banking systems," Ph.D. dissertation, Gjovik University College, 2012.

[11] A. Kumar and Y. Zhou, "Human identification using finger images," IEEE Transactions on Image Processing (TIP), vol. 21, no. 4, pp. 22282244, 2012.

[12] X. Xi, G. Yang, Y. Yin, and X. Meng, "Finger vein recognition with personalized feature selection," Sensors, vol. 13, no. 9, pp. $11243-$ $11259,2013$.

[13] M. S. Mohd Asaari, S. A. Suandi, and B. A. Rosdi, "Fusion of band limited phase only correlation and width centroid contour distance for finger based biometrics," Expert Systems with Applications, vol. 41, no. 7, pp. 3367-3382, 2014.

[14] Y. Yin, L. Liu, and X. Sun, "SDUMLA-HMT: a multimodal biometric database," in Biometric Recognition. Springer, 2011, pp. 260-268.

[15] C. Zhang, X. Li, Z. Liu, Q. Zhao, H. Xu, and F. Su, "The CFVD reflection-type finger-vein image database with evaluation baseline," in Biometric Recognition. Springer, 2013, pp. 282-287.

[16] E. C. Lee, H. C. Lee, and K. R. Park, "Finger vein recognition using minutia-based alignment and local binary pattern-based feature extraction," International Journal of Imaging Systems and Technology, vol. 19, no. 3, pp. 179-186, Sep 2009. [Online]. Available: http://dx.doi.org/10.1002/ima.v19:3

[17] N. Miura, A. Nagasaka, and T. Miyatake, "Extraction of finger-vein patterns using maximum curvature points in image profiles," IEICE Transaction on Information Systems, vol. E90-D, no. 8, pp. 1185-1194, Aug 2007.

[18] N. Miura, A. Nagassaka, and T. Miyatake, "Feature extraction of fingervein patterns based on repeated line tracking and its application to personal identification," Machine Vision and Applications, vol. 15, no. 4, pp. 194-203, Oct 2004.

[19] A. F. Martin, G. R. Doddington, T. Kamm, M. Ordowski, and M. A. Przybocki, "The DET curve in assessment of detection task performance." in EUROSPEECH, 1997. 